(eGFRcre). Shrunken pore syndrome (SPS) was defined as eGFRcys/eGFRcre $<60 \%$.

Results Comparing 20 patients with Cys $<1.2 \mathrm{mg} / \mathrm{L}$ and 32 patients with Cys $<1.2 \mathrm{mg} / \mathrm{L}$, SLE disease activity index (SLEDAI) was significantly higher in the former group than in the latter group $(7.7 \pm 7.8$ vs. $2.8 \pm 4.0, p<0.01)$, while the dose of corticosteroids did not differ significantly. The ratio of Cys $(\mathrm{mg} / \mathrm{L})$ to creatinine $(\mathrm{mg} / \mathrm{dl})$ was significantly higher in 25 patients with SLEDAI $\geq 3$ than in 27 patients with SLEDAI $<3(1.99 \pm 0.45$ vs. $1.68 \pm 0.35, \mathrm{p}<0.01)$. SLEDAI was significantly higher in 8 patients with SPS than in 44 patients without SPS $(10.6 \pm 10.9$ vs. $3.6 \pm 4.3, p<0.01)$. Organ involvement was found in $87.5 \%$ of SPS (2 with retinopathy, 1with cerebral haemorrhage, 1 with myositis plus nephritis, 1 with hemolytic anaemia, 1 with pleuritis, and 1 with enteritis), while 33 patients $(75.0 \%)$ without SPS were in stable state. Conclusions SLE patients with increased ratio of Cys to creatinine had high disease activity, and organ involvements were found with high frequency in patients with SPS.

\section{AN EVALUATION OF QUALITY OF LIFE IN AMBULATORY PATIENTS WITH SYSTEMIC LUPUS ERYTHEMATOSUS ATTENDING RHEUMATOLOGY CLINIC IN KENYATTA NATIONAL HOSPITAL}

1J Odhiambo*, ${ }^{2} \mathrm{GO}$ oyoo, ${ }^{2} \mathrm{E}$ Amayo. 'Nairobi, Kenya; ${ }^{2}$ University of Nairobi, Internal Medicine, Nairobi, Kenya

\subsection{6/lupus-2017-000215.453}

Background and aims Systemic Lupus Erythematosus (SLE) is a chronic autoimmune disease that affects the quality of life of those affected by it. The aim of this study is to document the quality of life of patients with SLE in Kenyatta National Hospital using LUPUS QOL questionnaire.

Methods Patients who satisfy the ACR (America College of Rheumatology) criteria were consecutively recruited. All patients with SLE attending the clinic were included in the study. Patients were examined for the presence of malar rash, discoid rash, arthritis/athralgia, photosensitivity, CNS symptoms, serositis and oral ulcers. The patients then filled the LUPUS QOL questionnaire. The data was then analysed using SPSS version 17.0 using student t test and regression analysis. Results Sixty two patients were analysed (60 females 2 males). Mean age of the population was 37.3 years ( $14-71$ years). Mean age at diagnosis was 34.5 years with mean duration of illness 1.5 years. Majority $(88.7 \%)$ had arthritis/athralgia, oral ulcers (62.9\%), malar rash (59.7\%), photosensitivity $(58.1 \%)$, serositis (32.3\%), CNS symptoms (27.4\%) and discoid rash (17.7\%). Patients scored globally low in all domains of LUPUS QOL. Highest domain was planning 63.7 (29.3), emotional health 61.3 (26.5), burden to others 58.9 (31.2), fatigue 57.5 (30.0), pain 56.6 (29.6), physical health 54.0 (23.3), body image 47.1 (24.2) intimate relations 41.1 (38.4).

Conclusions The HRQOL was low in all domains correlated with advance in age in the domains of physical health, burden to others, emotional health and fatigue.

\section{DISCORDANCE OF PATIENT AND PHYSICIAN HEALTH STATUS CONCERNS IN SYSTEMIC LUPUS ERYTHEMATOSUS}

1,2 JJY Ooi* ${ }^{2,3} \mathrm{~V}$ Golder, ${ }^{3} \mathrm{AS}$ Antony, ${ }^{3} \mathrm{~T}$ Ko, ${ }^{3} \mathrm{~S}$ Morton, ${ }^{3} \mathrm{R}$ Kandane-Rathnayake, ${ }^{3}$ EF Morand, ${ }^{3}$ AY Hoi. ' ${ }^{\prime}$ Alfred Health, Intern Program, Melbourne, Australia; ${ }^{2}$ Monash University, School of Clinical Sciences, Clayton, Australia; ${ }^{3}$ Monash Health, Department of Rheumatology, Clayton, Australia

\subsection{6/lupus-2017-000215.454}

Background and aims To investigate and compare the health status concerns of physicians and patients with systemic lupus erythematosus (SLE).

Methods Patients and their treating physicians completed a questionnaire asking their concern for specific disease manifestations and disease impact on quality of life. For each item, degree of concern was measured on a 5-point Likert scale. Sub-groups were compared by Kruskal-Wallis test for significance.

Results A total of 84 patients and 21 physicians participated. Patients were predominantly concerned with function and fatigue, whereas physicians focussed on organ complications of SLE. Seven out of the top 10 patient concerns showed statistically significant differences to physician ratings, including "reduced ability to perform activities of daily living (ADLs)" $(p=0.02)$ and "reduced ability to perform physical activities" $(p=0.04)$. All the top 10 physician concerns showed statistically significant differences to patient ratings, including "seizures" $(p=0.003)$ and "stroke" $(p=0.002)$. The top 3 patient concerns were routinely assessed by $47.6 \%, 42.9 \%$ and $9.5 \%$ of physicians, respectively.

Top 10 patient concerns:

Top 10 physician concerns:

Conclusions There was significant discordance between patient and physician health status concerns. Items which were ranked highly by patients were systematically underestimated by physicians, highlighting an urgent need to improve communication. Further studies could explore ways within the healthcare interaction that could improve patient satisfaction and disease control.

\section{VITAMIN D DEFICIENCY IS ASSOCIATED WITH INCEASED SERUM CHOLESTEROL AMONG PATIENTS WITH SYSTEMIC LUPUS ERYTHEMATOSUS}

${ }^{1} \mathrm{M}$ Petri ${ }^{*},{ }^{2} \mathrm{~L}$ Magder. 'Johns Hopkins University, Baltimore, USA; ${ }^{2}$ University of Maryland, Biostatistics, Baltimore, USA

\subsection{6/lupus-2017-000215.455}

Background and aims Vitamin D insufficiency/deficiency is common in SLE. In other populations, Vitamin D has been associated with cardiovascular risk factors such as blood pressure and serum cholesterol. We assessed whether there was an association between serum Vitamin D and total serum cholesterol in a large SLE cohort.

Methods Serum 25-hydroxy vitamin D [25(OH)D] was measured at quarterly clinic visits in a large SLE cohort. 1358 different patients were observed from 1 to 40 visits (the median was 11). The patients were $92 \%$ female, 50\% Caucasian, $41 \%$ 